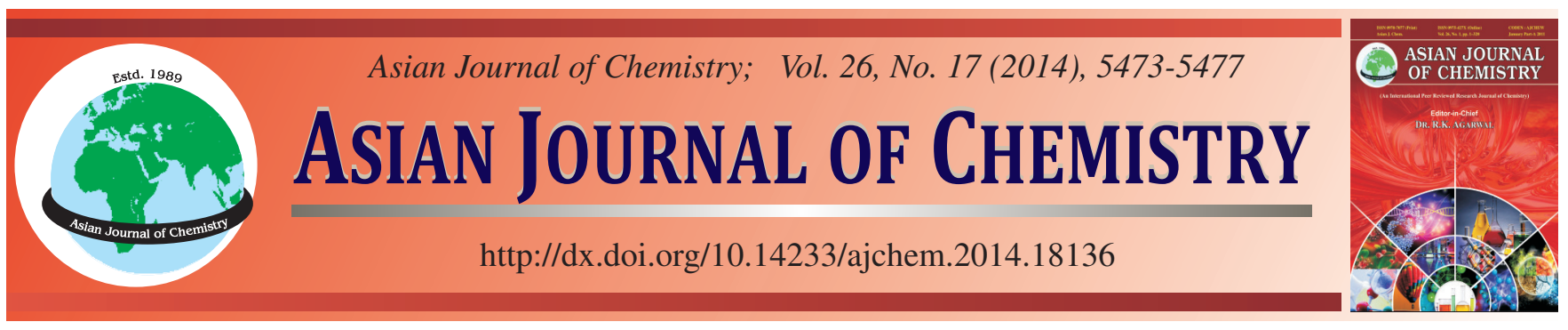

\title{
Magnetic Properties of Manganese-Iron Multi-Metal Cyanide Compound $\mathrm{Ni}_{1.15} \mathrm{Mn}_{0.35}\left[\mathrm{Fe}(\mathrm{CN})_{6}\right] \cdot 6 \mathrm{H}_{2} \mathrm{O} \dagger$
}

\author{
Yun HE ${ }^{1}$, Zhimin Ji ${ }^{1}$, Qing Lin ${ }^{1,2, *}$, Haifu HuAng ${ }^{1,3}$ and Wenjie Kong ${ }^{1}$
}

${ }^{1}$ College of Physics and Technology, Guangxi Normal University, Guilin 541004, P.R. China

${ }^{2}$ Department of Information Technology, Hainan Medical College, Haikou 571101, P.R. China

${ }^{3}$ Department of Physics, Nanjing University, Nanjing 210093, P.R. China

*Corresponding author: Fax:+86 773 5840785; Tel: +86 773 5811173; E-mail: hy@gxnu.edu.cn

\begin{abstract}
A multi-metal prussian blue compound $\mathrm{Ni}_{1.15} \mathrm{Mn}_{0.35}\left[\mathrm{Fe}(\mathrm{CN})_{6}\right] \cdot 6 \mathrm{H}_{2} \mathrm{O}$ has been synthesized. The IR spectrum of the compound shows two bands at 2096.42 and $2162.13 \mathrm{~cm}^{-1}$ indicating the existence of two types of cyanide groups.It undergoes a paramagnetic to ferromagnetic transition at $21 \mathrm{~K}$. These results indicate that there exist a ferromagnetic exchange interaction in the complexes. According to Curie-Weiss law,paramagnetic Curie temperature $(\Theta)$ is $13.81 \mathrm{~K}$ and the Curie-Weiss constant $(C)$ is $2.21 \mathrm{~cm}^{3} \mathrm{~K} \mathrm{~mol}^{-1}$. In addition, the $\chi^{\prime}(\mathrm{T})$ and $\chi^{\prime \prime}(\mathrm{T})$ show clear peak around $18-20 \mathrm{~K}$. The behaviour of $\chi^{\prime}$ and $\chi$ " is typical of a spin glass state go through a maximum with strong frequency dependence. The observed value of coercive field $\left(\mathrm{H}_{\mathrm{c}}\right)$ and remanent magnetization $\left(\mathrm{M}_{\mathrm{r}}\right)$ for the compound are $1.72 \mathrm{KOe}$ and $0.682 \mu_{\beta}$, respectively.
\end{abstract}

Keywords: Prussian blue analogue, Magnetic transition, Infrared spectroscopy, Ferromagnetic, Spin-glass.

ᄂ - - - - - - - - - - - - - - - - - - - - - - - - - -

\section{INTRODUCTION}

Recently, molecule-based magnet have been investigated as new magnetic materials $\mathrm{s}^{1-3}$. In that field cyano-bridged complexes play an important role due to their special structure and outstanding magnetic properties as molecule-based magnets. Prussian blue analogues are the most known and studied cyanide-based frameworks ${ }^{3-6}$. Prussian blue analogues $\mathrm{C}_{n} \mathrm{~A}_{P}\left[\mathrm{~B}(\mathrm{CN})_{6}\right]_{q} \cdot \mathrm{xH}_{2} \mathrm{O}$ (Fig. 1) show various magnetic properties depending on their transition metal ion ${ }^{4-7}$. The Mn-Fe cyanides, whose chemical formula is $\mathrm{A}_{\mathrm{x}} \mathrm{Mn}\left[\mathrm{Fe}(\mathrm{CN})_{6}\right]_{\mathrm{y}} \cdot \mathrm{zH}_{2} \mathrm{O}(\mathrm{A}=\mathrm{K}$, $\mathrm{Ni}, \mathrm{Cs}, \mathrm{Cu}, \mathrm{Co}$ ), have been attracting renewed interest of the material scientists. The appeal of Prussian blue itself lies both in its optical and magnetic properties. The substitution of Fe(II) and $\mathrm{Fe}$ (III) by other paramagnetic metal ions $\mathrm{M}$ and $\mathrm{M}$ ' has afforded a series of Prussian blue analogues with face-centered cubic structures. Hashimoto et al. ${ }^{4,5}$ have prepared a series of molecular alloy magnet, which magnetic parameters like saturation magnetization $\left(\mathrm{M}_{\mathrm{s}}\right)$, Weiss paramagnetic Curie temperature $(\Theta)$, coercive field $\left(\mathrm{H}_{\mathrm{c}}\right)$, transition temperature $\left(\mathrm{T}_{\mathrm{c}}\right)$, etc. In this context, we have prepared multi-metal compound $\mathrm{Ni}_{1.15} \mathrm{Mn}_{0.35}\left[\mathrm{Fe}(\mathrm{CN})_{6}\right] \cdot 6 \mathrm{H}_{2} \mathrm{O}$ by co-precipitation method and the magnetic properties have been studied.

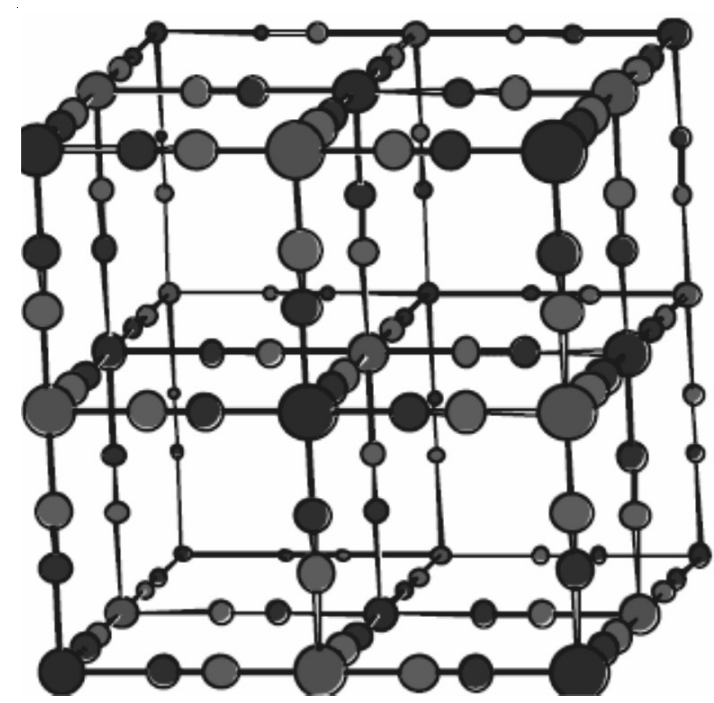

Fig. 1. Structure of Prussian blue analog $\mathrm{A}_{\mathrm{P}}\left[\mathrm{B}(\mathrm{CN})_{6}\right]_{\mathrm{q}} \cdot \mathrm{xH}_{2} \mathrm{O}$

\section{EXPERIMENTAL}

$\mathrm{NiCl}_{2} \cdot 6 \mathrm{H}_{2} \mathrm{O}, \mathrm{Mn}\left(\mathrm{SO}_{4}\right)_{2} \cdot 6 \mathrm{H}_{2} \mathrm{O}$ and $\mathrm{K}_{3} \mathrm{Fe}(\mathrm{CN})_{6}$ are reagent grade and without further purification. Elemental analysis $(\mathrm{C}$, 
N, H) were performed on Perk-Elmer 2400 II analyzer. IR spectrum were recorded on a Perkin-Elmer FT-IR spectrophotometer as $\mathrm{KBr}$ pellet in the $4000-400 \mathrm{~cm}^{-1}$ range. Thermal gravimetric analysis (TG) were performed on Perkin Elmer TGA detector. Magnetization measurements were measured on a Quantum Design MPMS-7 magnetometer in the range of 2-300 K.

Synthesis of $\mathrm{Ni}_{1.15} \mathrm{Mn}_{0.35}\left[\mathrm{Fe}(\mathrm{CN})_{6}\right] \cdot \mathbf{6} \mathrm{H}_{2} \mathrm{O}$ : Polycrystalline compound $\mathrm{Ni}_{1.15} \mathrm{Mn}_{0.35}\left[\mathrm{Fe}(\mathrm{CN})_{6}\right] \cdot 6 \mathrm{H}_{2} \mathrm{O}$ was prepared by coprecipitation method. To a solution of $150 \mathrm{~mL} \mathrm{NiCl} 2 \cdot 6 \mathrm{H}_{2} \mathrm{O}$ $(2.3 \mathrm{mmol})$ was mixed $150 \mathrm{~mL} \mathrm{MnSO}_{4} \cdot 6 \mathrm{H}_{2} \mathrm{O}(0.7 \mathrm{mmol})$ solution. Then a solution of $\mathrm{K}_{3} \mathrm{Fe}(\mathrm{CN})_{6}(2 \mathrm{mmol})$ in water (100 $\mathrm{mL}$ ) was slowly added to the mixed solution of $\mathrm{NiCl}_{2} \cdot 6 \mathrm{H}_{2} \mathrm{O}$ and $\mathrm{MnSO}_{4} \cdot 6 \mathrm{H}_{2} \mathrm{O}$ and a solid was precipitated immediately. After $48 \mathrm{~h}$, the precipitates were filtered, washed repeatedly with water and dried at $45^{\circ} \mathrm{C}$. Elemental analysis to measure C, N, H mass ratio: found (\%): C, 17.24; N, 20.38; H, 3.26; calcd. (\%): C, 17.71; N, 20.66; H, 2.97. Thermal gravimetric analysis (TG) has given weight ratio of the crystal water in molecular formula weight, the compound contains 6 crystal waters by calculation.

IR spectrum: Prussian blue analogs have face-centered cubic lattice in which two transition metals ions are interconnected via $\mathrm{C}-\mathrm{N}$ bridges (cyanometalate-based systems) ${ }^{6,7}$. Cyano compounds are easily identified since exhibit sharp band stretching, the IR spectrum of the compound $\mathrm{Ni}_{1.15} \mathrm{Mn}_{0.35}\left[\mathrm{Fe}(\mathrm{CN})_{6}\right] \cdot 6 \mathrm{H}_{2} \mathrm{O}$ shows two bands at 2096.42 and $2162.13 \mathrm{~cm}^{-1}$ indicating the existence of two types of cyanide groups (Fig. 2). Compounds are easily identified by their stretching frequencies in 2200$2000 \mathrm{~cm}^{-1}$ range, which are consistent with the formation of bridging cyanide groups and there are two different coordination environment. Compared to the compound $\mathrm{K}_{3} \mathrm{Fe}(\mathrm{CN})_{6}$ $\left(v_{\mathrm{CN}}=2121.29 \mathrm{~cm}^{-1}\right)$, which may due to the change in the spin states and valence states of metal ions. The broad peak at 3432 and $1615.02 \mathrm{~cm}^{-1}$ are assigned to the $v(\mathrm{O}-\mathrm{H})$ of the crystal water stretching vibrations. The alkali metal cations are interstitial in the lattice and the water molecules substitute the $\mathrm{C}-\mathrm{N}$ groups at the missing $\mathrm{Fe}(\mathrm{CN})_{6}$ sites.

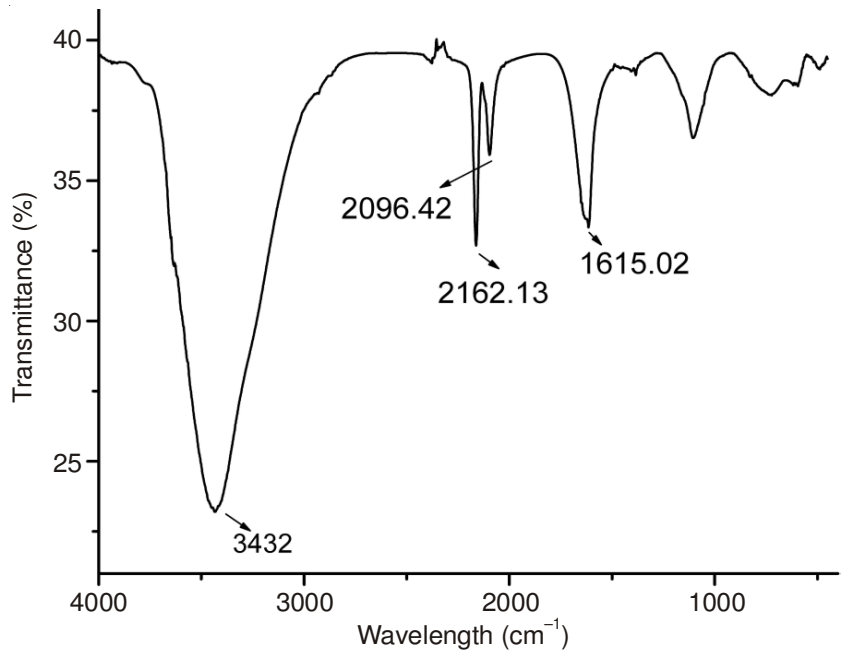

Fig. 2. FT-IR spectrum of the compound

\section{RESULTS AND DISCUSSION}

DC magnetic susceptibility: The magnetic susceptibility of the compound $\mathrm{Ni}_{1.15} \mathrm{Mn}_{0.35}\left[\mathrm{Fe}(\mathrm{CN})_{6}\right] \cdot 6 \mathrm{H}_{2} \mathrm{O}$ was measured from 2-300 K (Fig. 3), which corresponds to the steepest rise of magnetization with decreasing temperature. A magetic transition temperature $\left(\mathrm{T}_{\mathrm{c}}\right)$ of $21 \mathrm{~K}$ is observed by minima of $\mathrm{dM} / \mathrm{dT}$ vs. T curve (Fig. 4) and lower than that for the compound $\mathrm{Ni}_{1.5}\left[\mathrm{Fe}(\mathrm{CN})_{6]} \cdot \mathrm{xH}_{2} \mathrm{O}\left(\mathrm{T}_{\mathrm{C}}=23.6 \mathrm{~K}\right)^{8}\right.$.

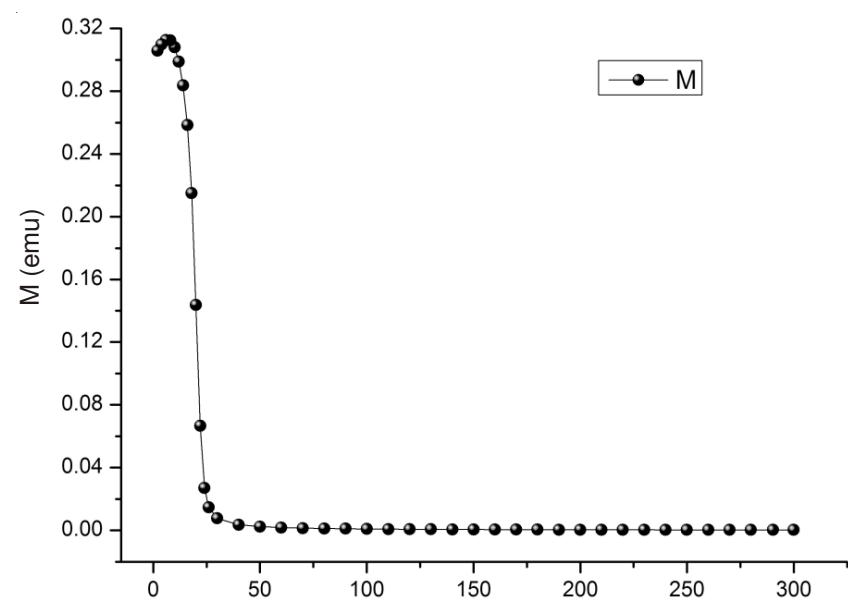

Fig. 3. Temperature dependence of magnet for the compound

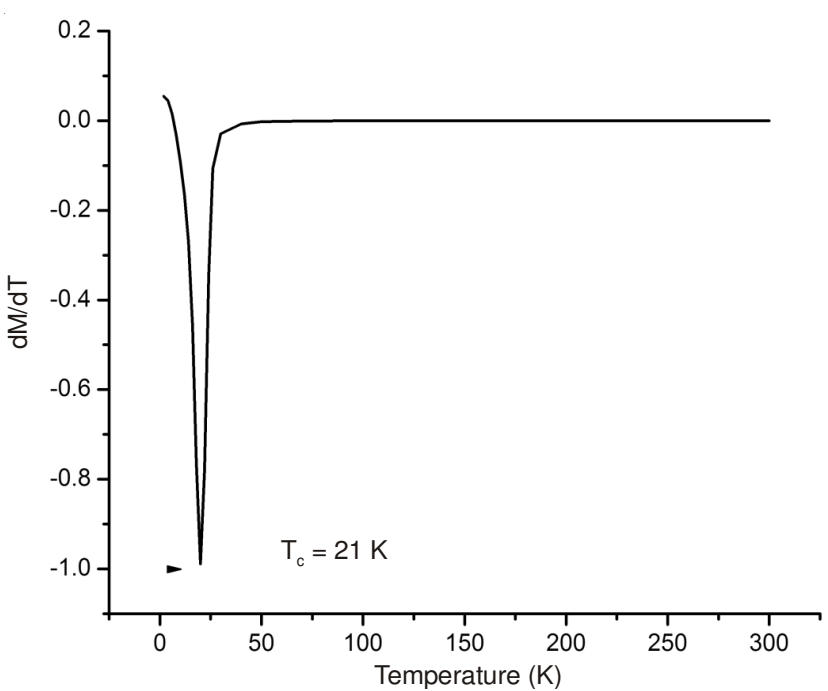

Fig. 4. dM/dT versus $\mathrm{T}$ for the compound

The inverse susceptibility as a function of temperature in the paramagnetic state is shown in Fig. 5, the values of $\chi_{\mathrm{m}}$ gradually increase and then sharply increase after $25 \mathrm{~K}$ with a further decrease of the temperature. The $\chi_{\mathrm{m}}$ shows a sharp maximum at $7 \mathrm{~K}$ which is a characteristic of a ferromagnet ${ }^{9-11}$. According to $\chi_{\mathrm{m}}{ }^{-1}$ versus $\mathrm{T}$ curve (Fig. 6), the $\chi_{\mathrm{m}}{ }^{-1}$ vs. T above $30 \mathrm{~K}$ obey the Curie-Weiss law ${ }^{12-14}$ with a Curie constant of $\mathrm{C}$ $=2.21 \mathrm{~cm}^{3} \mathrm{~K} \mathrm{~mol}^{-1}$ and Weiss paramagnetic Curie temperature of $\Theta=13.81 \mathrm{~K}$. The values of $\mathrm{T}_{\mathrm{c}}, \Theta$ and $\mathrm{C}$ are different from those values for ferrimagnet $\mathrm{Ni}_{1.5}\left[\mathrm{Fe}(\mathrm{CN})_{6]} \times \mathrm{XH}_{2} \mathrm{O}^{8}\right.$ and $\mathrm{Mn}_{3}\left[\mathrm{Fe}(\mathrm{CN})_{6}\right]_{2} \cdot 15 \mathrm{H}_{2} \mathrm{O}^{10}$.

The magnetic properties of compound $\mathrm{Ni}_{1.15} \mathrm{Mn}_{0.35}\left[\mathrm{Fe}(\mathrm{CN})_{6}\right] \cdot 6 \mathrm{H}_{2} \mathrm{O}$ under the form of $\chi_{\mathrm{m}} \mathrm{T} v$ s. $\mathrm{T}$ plot are shown in the Fig. 7 , the $\chi_{\mathrm{m}} \mathrm{T}$ at room temperature is 2.57 $\mathrm{cm}^{3} \mathrm{~K} \mathrm{~mol}^{-1}$. Upon cooling, $\chi_{\mathrm{m}} \mathrm{T}$ increases smoothly until $25 \mathrm{~K}$ and then it undergoes a sharp increase to reach a value of 111 $\mathrm{cm}^{3} \mathrm{~K} \mathrm{~mol}^{-1}$ at $15 \mathrm{~K}$ and final decrease at lower temperature, indicating antiferromagnetic interaction ${ }^{12-14}$. A curve of $\mu_{\text {eff }} v s$. 


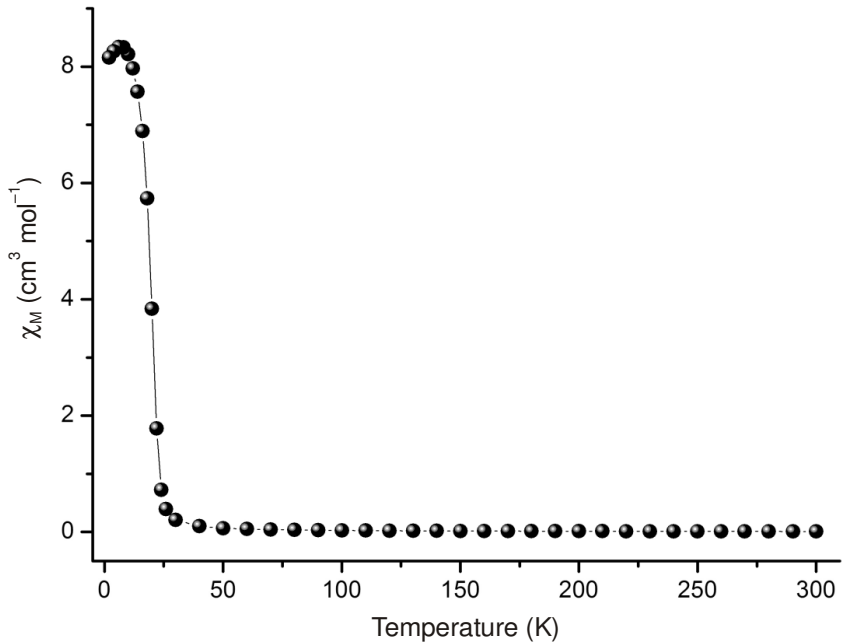

Fig. 5. Temperature dependence of $\chi_{\mathrm{m}}$ for the compound

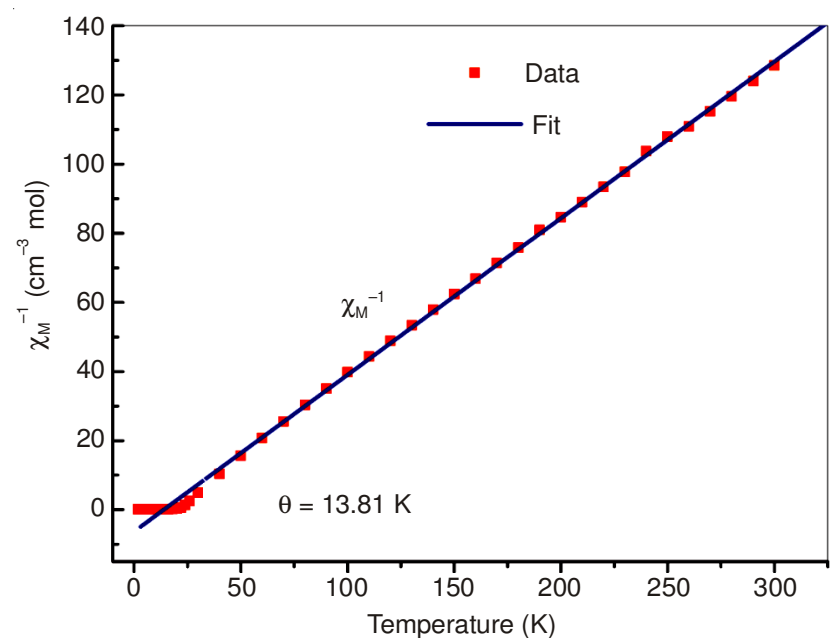

Fig. 6. $\chi_{\mathrm{m}}^{-1}$ versus $\mathrm{T}$ for the compound

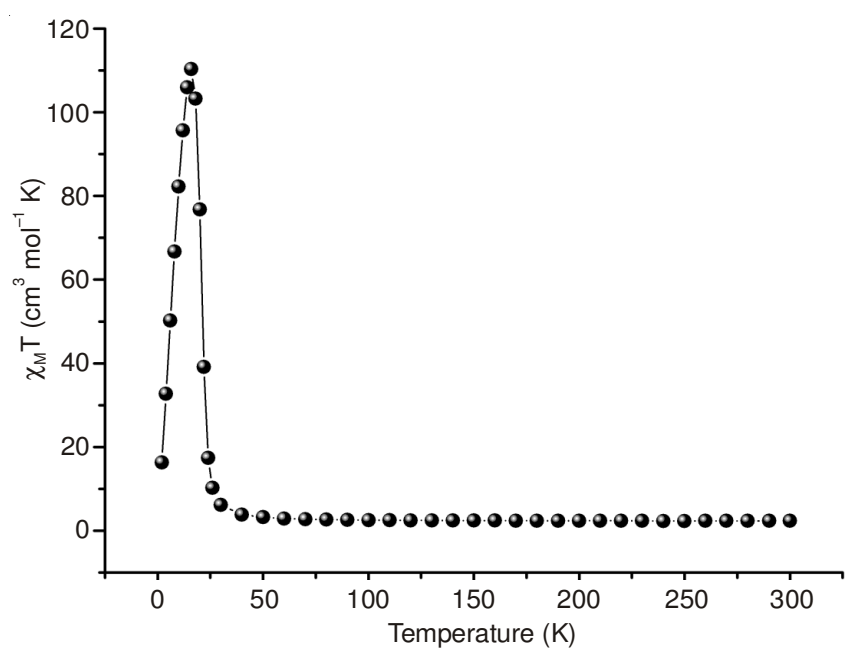

Fig. 7. $\chi_{\mathrm{m}} \mathrm{T}$ versus $\mathrm{T}$ plot of the compound

$\mathrm{T}$ is shown in Fig. 8 , the $\mu_{\text {eff }}$ value at $300 \mathrm{~K}$ is $4.32 \mu_{\beta}$. The effective moment $\mu_{\text {eff }}$ sharply increase to reach maximum of $29.71 \mu_{\beta}$ at $15 \mathrm{~K}$ and final decrease at lower temperature, indicating antiferromagnetic interaction between paramagnetic centers. The behaviour is a characteristic of a ferrimagnet ${ }^{15-17}$.

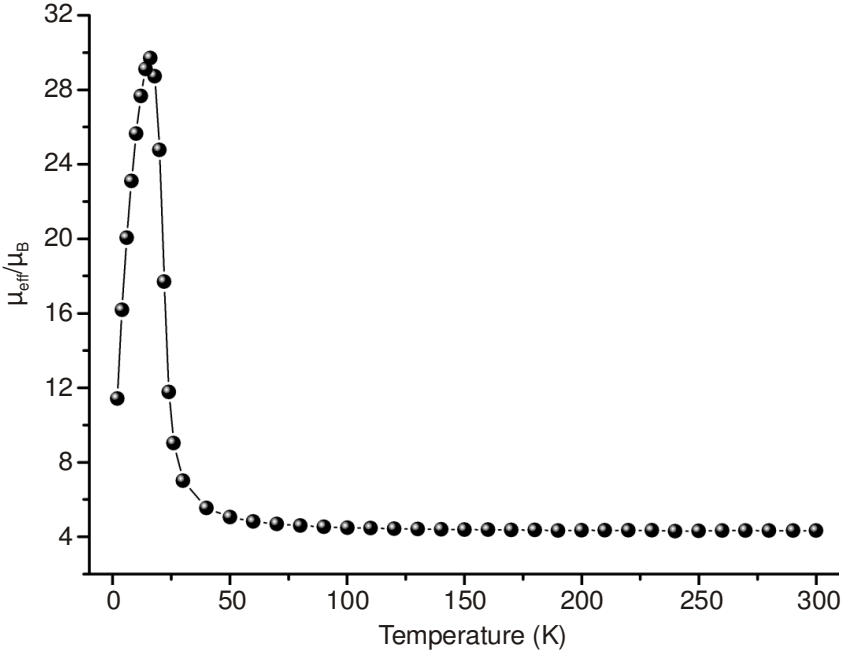

Fig. 8. $\mu_{\text {eff }}$ versus $\mathrm{T}$ plot of the compound

AC magnetic susceptibility: The magnetic susceptibility in the presence of an oscillating AC magnetic field at different frequencies (100-997 Hz) has been measured. Alternating current $(\mathrm{AC})$ measurements allow probing the relaxation time of the magnetization. When the relaxation time of the magnetization of the system probed and the frequency of the oscillating field become the same order of magnitude, the magnetization does not follow the magnetic field and the susceptibility will exhibit two components: the real in-phase part $\left(\chi^{\prime}\right)$ and the imaginary out of phase part $\left(\chi^{\prime \prime}\right)$. It is also confirmed that there exist a spin-glass behaviour in the compound $\mathrm{Ni}_{1.15} \mathrm{Mn}_{0.35}\left[\mathrm{Fe}(\mathrm{CN})_{6}\right] \cdot 6 \mathrm{H}_{2} \mathrm{O}$ through $\mathrm{AC}$ magnetization curves $^{18-20}$, the $\chi$ ' and $\chi$ " values were measured at 4 Oe AC amplitude as shown in Fig. 9. The AC magnetic susceptibilities show that the in-phase component $\left(\chi^{\prime}\right.$, real) has a maximum at about $20 \mathrm{~K}$ for frequencies (at 100, 400, 800, $997 \mathrm{~Hz}$ ) and that a significant out-of-phase component ( $\chi$ ", imaginary) appears, confirming the long-range ferromagnetic ordering. On decreasing temperature, the $\chi$ ' behave: increase abruptly at around $25 \mathrm{~K}$, reach the maximum at about $20 \mathrm{~K}$ and then decrease slowly toward zero. The $\chi$ " increases to the maximum around $18-20 \mathrm{~K}$. The AC susceptibility measurements for the compound also confirm the magnetic phase transition in both complexes, showing a peak in $\chi$ ' signal and $\chi$ " signal that is non-zero below $25 \mathrm{~K}$. Surprisingly, both $\chi$ ' and $\chi$ ", go through a maximum with strong frequency dependence. While for the real component the intensity of the peaks increase with decreasing frequencies, in the imaginary component the peaks decrease with decreasing frequencies. This behaviour of $\chi$ ' and $\chi$ " is typical of a spin glass state ${ }^{21,22}$. In fact, the temperature value of the maximum of $\chi$ at a given frequency corresponds to the blocking temperature $\left(\mathrm{T}_{\mathrm{N}}=\mathrm{T}_{\max }\right)$ and the peak temperature $T_{f}(w)$ shifts toward higher temperatures with increasing frequency (Fig. 10) because frequency dependent shoulders are readily apparent. Measurements at different frequencies show that the maxima of $\chi^{\prime}$ and $\chi^{\prime \prime}$ are strongly frequency dependent and shift to low temperature when the frequency of the oscillating field is decreased confirming the slow relaxation of the magnetization. The in-phase and out-of-phase peak maxima are both shifted to the direction of higher tempe- 


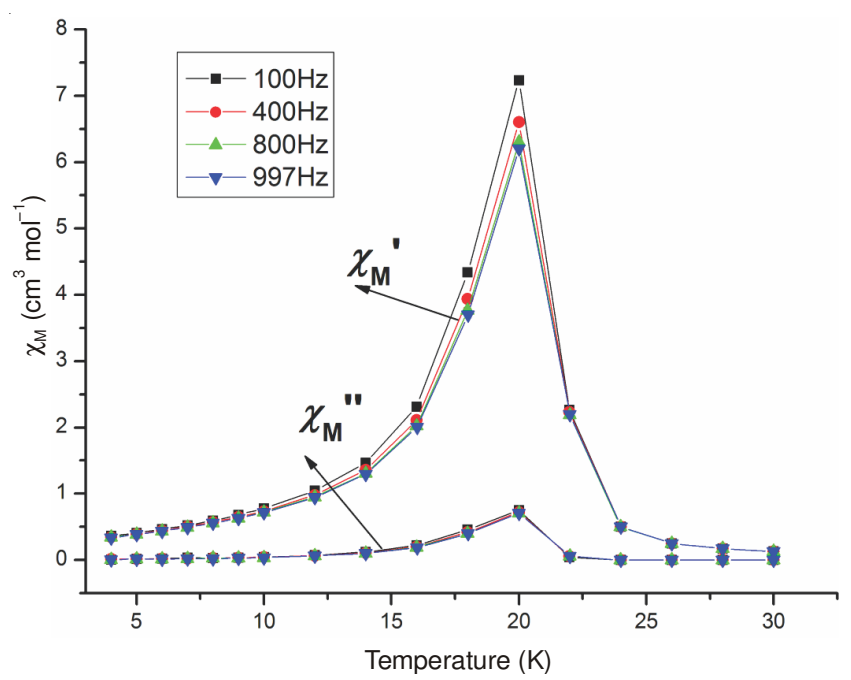

Fig. 9. $\chi^{\prime}(\mathrm{T})$ and $\chi^{\prime \prime}(\mathrm{T})$ curves of the compound

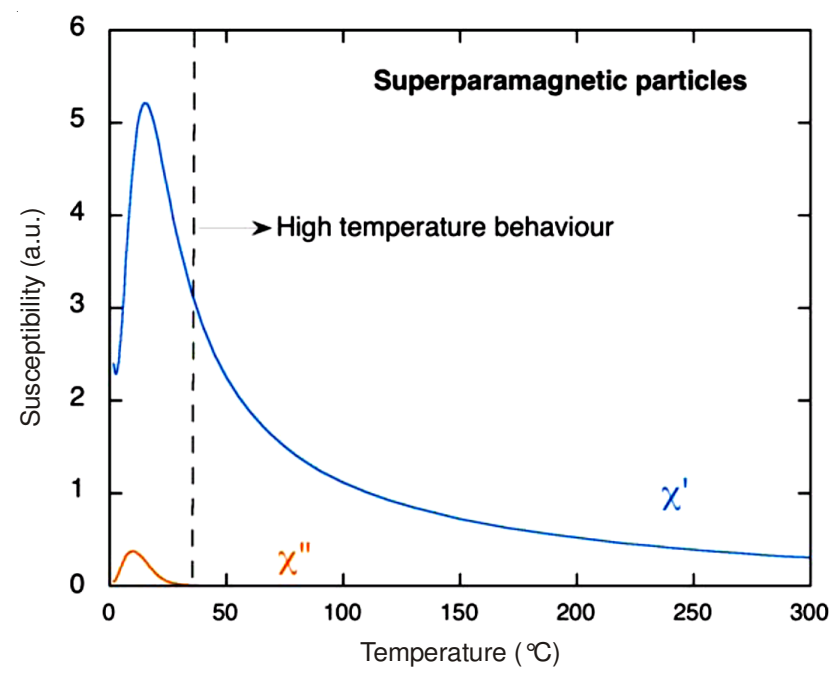

Fig. 10. Scheme of temperature dependence of $\chi(T)$

rature, respectively. The peak temperature shifts toward higher temperatures with increasing frequency.

Field-dependent of magnetization and hysteresis behaviour: The magnetic behaviour is further characterized by the measurements of hysteresis behaviour and field-dependent magnetization (Fig. 11). The observed $M_{s}$ value is $2.05 \mu_{\beta}$ at $50 \mathrm{kOe}$, but the compound does not reach full saturation.

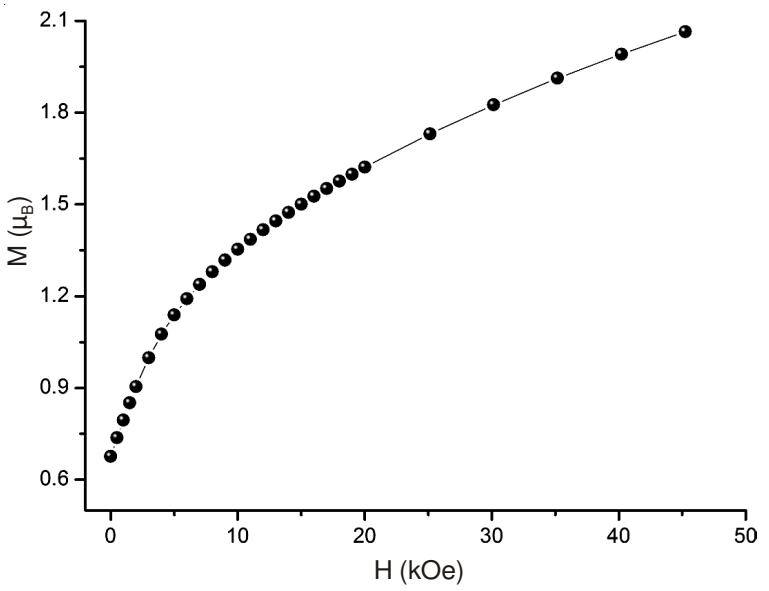

Fig. 11. Field-dependent magnetization curves
The hysteresis curves measured at $4 \mathrm{~K}$, shown in the Fig. 12. The coercive field $\left(\mathrm{H}_{\mathrm{c}}\right)$ value of $1.72 \mathrm{kOe}$ and remanent magnetization $\left(\mathrm{M}_{\mathrm{r}}\right)$ value of $0.682 \mu_{\beta}$ for the compound compound $\mathrm{Ni}_{1.15} \mathrm{Mn}_{0.35}\left[\mathrm{Fe}(\mathrm{CN})_{6}\right] \cdot 6 \mathrm{H}_{2} \mathrm{O}$. The observed value of $\mathrm{H}_{\mathrm{c}}$ is different from many other hexacyano analogues? Magnetic parameters like saturation magnetization $\left(M_{s}\right)$, coercive field (Hc), Curie constant (C), Weiss paramagnetic Curie temperature $(\Theta)$, transition temperature $\left(T_{c}\right)$, are different from the those of the bimetallic cyanide-bridged compounds $\mathrm{Ni}_{1.5}\left[\mathrm{Fe}(\mathrm{CN})_{6]} \cdot \mathrm{xH}_{2} \mathrm{O}^{8}\right.$ and $\mathrm{Mn}_{3}\left[\mathrm{Fe}(\mathrm{CN})_{6}\right]_{2} \cdot 15 \mathrm{H}_{2} \mathrm{O}^{10}$. The alkali metal cations are interstitial in the lattice and the water molecules substitute the missing $\mathrm{C}-\mathrm{N}$ groups at the missing $\mathrm{Fe}(\mathrm{CN})_{6}$ sites $^{21,22}$.

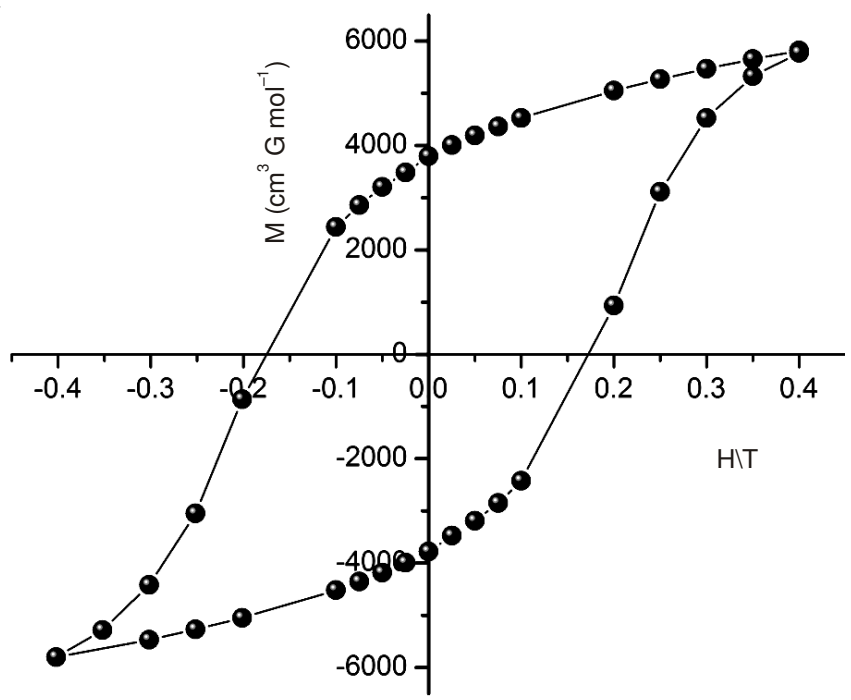

Fig. 12. Hystersis loop for the compound

\section{Conclusion}

In the present work, we have reported the spin-glass behaviour in the multi-metallic compound $\mathrm{Ni}_{1.15} \mathrm{Mn}_{0.35}\left[\mathrm{Fe}(\mathrm{CN})_{6}\right] \cdot 6 \mathrm{H}_{2} \mathrm{O}$. The IR spectrum shows two bands at 2096.42 and 2162.13 $\mathrm{cm}^{-1}$ indicating the existence of two types of cyanide groups. The Curie-Weiss constants are $\mathrm{C}=2.21 \mathrm{~cm}^{3} \mathrm{~K} \mathrm{~mol}^{-1}, \Theta=$ $13.81 \mathrm{~K}$. It undergoes a paramagnetic to ferromagnetic transition at $21 \mathrm{~K}$. These results indicate that there exist a ferromagnetic exchange interaction in the complexes. This behaviour of $\chi$ ' and $\chi$ " is typical of a spin glass state go through a maximum with strong frequency dependence. The observed value of coercive field $\left(\mathrm{H}_{\mathrm{c}}\right)$ and remanent magnetization $\left(\mathrm{M}_{\mathrm{r}}\right)$ for the compound are $1.72 \mathrm{KOe}$ and $0.682 \mu_{\beta}$, respectively. The values of $\mathrm{T}_{c}, \Theta$ and $\mathrm{C}$ are different from those values for ferrimagnet $\mathrm{Ni}_{1.5}\left[\mathrm{Fe}(\mathrm{CN})_{6}\right] \cdot \mathrm{xH}_{2} \mathrm{O}$ and $\mathrm{Mn}_{3}\left[\mathrm{Fe}(\mathrm{CN})_{6}\right]_{2} \cdot 15 \mathrm{H}_{2} \mathrm{O}$. Therefore, synthesis ideas of molecular alloy magnet can be regarded as a synthesis method to expand a new type of magnetic functional materials, which magnetic properties can be controlled by changing the different transition metal cations.

\section{ACKNOWLEDGEMENTS}

This work was financially supported by the National Natural Science Foundation of China (No. 11164002, 11364004); NSF of Guangxi Province (No. 0991092). 


\section{REFERENCES}

1. S. Ohkoshi, T. Iyoda, A. Fujishima and K. Hashimoto, Phys. Rev. B, 56, 11642 (1997).

2. O. Sato, Accounts Chem. Res., 36, 692 (2003).

3. S. Ohkoshi, O. Sato, T. Iyoda, A. Fujishima and K. Hashimoto, Inorg. Chem., 36, 268 (1997).

4. S. Ohkoshi, T. Hozumi and K. Hashimoto, Phys. Rev. B, 64, 132404 (2001)

5. S. Ohkoshi, Y. Abe, A. Fujishima and K. Hashimoto, Rev. Lett., 82, 1285 (1999).

6. V. Gadet, T. Mallah, I. Castro, M. Verdaguer and P. Veillet, J. Am. Chem. Soc., 114, 9213 (1992).

7. M. Verdaguer and G. Girolami, Magnetic Prussian Blue Analogs, WileyVCH Verlag GmbH and Co. (2004)

8. S. Juszczyk, C. Johansson, M. Hanson, A. Ratuszna and G. Malecki, J. Phys.: Condens. Matter., 6, 5697 (1994).

9. S. Ferlay, T. Mallah, R. Ouahès, P. Veillet and M. Verdaguer, Nature, 378, 701 (1995).
10. A. Kumar and S.M. Yusuf, Physica B, 362, 278 (2005).

11. D. Gatteschi, O. Kahn, J.S. Miller and F. Palacio, NATO ASI Series E: Applied Science, p. 198 (1991).

12. D. Zhang, Z. Zhao and X. Chen, Asian J. Chem., 25, 3509 (2013).

13. O. Sato, Y. Einapa, A. Fujishima and K. Hashimoto, Inorg. Chem., 38, 4405 (1999).

14. M. Ohba and H. Okawa, Coord. Chem. Rev., 198, 313 (2000).

15. A. Kumar, S.M. Yusuf and L. Keller, Physica B, 385-386, 444 (2006).

16. J.S. Miller, A.J. Epstein and W.M. Reiff, Chem. Rev., 88, 201 (1988).

17. S.M. Holmes and G.S. Girolami, J. Am. Chem. Soc., 121, 5593 (1999).

18. J.S. Miller and A.J. Epstein, Angew. Chem. Int. Ed. Engl., 33, 385 (1994).

19. S. Ohkoshi and K. Hashimoto, Phys. Rev. B, 60, 12820 (1999).

20. D. Sherrington and S. Kirkpatrick, Phys. Rev. Lett., 35, 1792 (1975).

21. A. Kumar, S.M. Yusuf and L. Keller, Phys. Rev. B, 71, 054414 (2005).

22. K. Binder and A.P. Young, Rev. Mod. Phys., 58, 801 (1986). 